\title{
Confusions Regarding Quantum Mechanics
}

\author{
Gerard 't Hooft, reply by Sheldon Lee Glashow
}

In response to "The Yang-Mills Model" (Vol. 5, No. 2).

To the editors:

Quantum mechanics was one of the most significant and important discoveries of twentieth-century science. It all began, I think, in the year 1900 when Max Planck published his paper entitled "On the Theory of the Energy Distribution Law of the Normal Spectrum." In it, he describes a simple observation: if one attaches an entropy to the radiation field as if its total energy came in packages-now called quanta - then the intensity of the radiation associated to a certain temperature agrees quite well with the observations. Planck had described his hypothesis as "an act of desperation."2 But it was the only one that worked. Until that time, the best attempt at performing such a calculation had resulted in Wien's law. This was an important result at the time, but only worked at the lowest frequencies of the radiation, failing bitterly at high frequencies and smaller wavelengths. Wien's law would yield a badly divergent, hence meaningless, expression for the intensity of the radiation emitted at high frequencies.

The history of what happened next is well known and has been recounted in excellent reports, which, I think, do not need to be repeated here. ${ }^{3}$ Physicists realized that all oscillatory motion apparently comes in energy packets that seem to behave as particles, and that the converse also seems to be true: all particles with definite energies must be associated to waves. This all culminated in the theory of quantum mechanics. The year 1926 provided a new landmark: Erwin Schrödinger's equation. ${ }^{4}$ The title of my essay is not intended to cast doubt on this equation; the Schrödinger equation has been and is still the pivotal equation on which much of physics and all of chemistry is based, and I am in awe of it just as many researchers before me have been.

In his original paper, Schrödinger went quite far in discussing Hamilton's principle, boundary conditions, the hydrogen atom, and the electromagnetic transitions from one energy level to the next. The confusions to which I refer in my title arose when more and more researchers began to argue about how the equation is supposed to be interpreted. Why is it that positions and velocities of particles at one given moment cannot be calculated, or even defined unambiguously?

Physicists know very well how to use the equation. They use it to derive with perplexing accuracy the properties of atoms, molecules, elementary particles, and the forces between all of these. The way the equation is used is nothing to complain about, but what exactly does it say?

The first question one may rightfully ask, and that has been asked by many researchers and their students, is this:

What do these wave functions represent? In particular, what is represented by the wave functions that are not associated to photons, the energy packets of the electromagnetic field, which we think we understand very well? What do those waves stand for that are associated to electrons, or other elementary particles, or even molecules and larger things, including cats and eventually physicists? What happens to its wave function when the particle is actually observed?

One extremely useful observation was made by Max Born: the absolute square of a wave function, at some spot in position space, must simply stand for the probability of finding the particle there. ${ }^{5}$ This made sense, and it was rightly adopted as a useful recipe for dealing with this equation. But then many more questions were asked, many of them well posed, but the answers sounded too ridiculous to be true, and, as I shall try to elucidate, they are too ridiculous to be true. The truth is much simpler to grasp, even if the mathematics needed to back up the concepts can be delicate. This is astonishing. Almost a full century has passed since the equation was written, and fierce discussions have been held; quite a few standpoints were vigorously defended and equally vigorously attacked. We still do not know what or whom to believe, but it still goes on, while others get irritated by this display of impotence. ${ }^{6}$ Why is it that we still do not agree? I think I know the answers, but almost everyone disagrees with me. 
In fact, one possible reply is that one could decide to ignore the question. Paul Dirac, for instance, advised not to ask questions that cannot be answered by experiment. Such questions are of secondary importance. We know precisely how to use Schrödinger's equation; all that scientists have to do is insert the masses and coupling parameters of all known particles into the equation, and calculate. What else can you ask for? Many of my colleagues decided to be strictly agnostic about the interpretation, which is as comfortable a position to take as it was for nineteenth-century scientists to stay agnostic about the existence of atoms.

But yes indeed, there is something else. What are those masses and coupling strengths? Do particles exist that physicists have not yet been able to detect? Isn't it the scientist's job to make predictions about things they have not yet been able to unravel? This is a question that is haunting us physicists. We have arrived at a splendid theory that accounts for almost anything that could be observed experimentally. It is called the Standard Model of the subatomic particles. But this model also says that particles and forces may exist that physicists cannot detect today. Can we produce any theory that suggests what one might be able to find, in some distant future? And as of all those particles and forces that we do know about, is there a theory that explains all their details?

Today's theories give us little to hang on to. This is why it is so important to extend as far as we can our abilities to do experiments. Audacious plans have been unfolded recently by the European particle physics laboratory CERN, for building a successor to its highly successful Large Hadron Collider (LHC). While the LHC has provided strong evidence supporting the validity of the Standard Model up to the tera-electron volts $(\mathrm{TeV})$ domain, theoreticians find it more and more difficult to understand why this model would be all there is to describe what happens beyond that scale. There must be more, but our theoretical reasoning leads to more questions calling into doubt the extent to which this model can be regarded as natural when more of the same particles with higher energies are included, while the existence of totally new particles would be denied.

Inspired by what historians of science report about similar situations in the past history of our field, investigators are hoping for a paradigm shift. While it is easy to postulate that we are doing something wrong, most suggestions for improvement are futile; suggesting that the Standard Model might be wrong is clearly not going to help. The Future Circular Collider, proposed by CERN, is a much better idea. It will be an accelerator with a circumference of about 100 kilometers, able to reach a center-of-mass collision energy of $100 \mathrm{TeV}$. The importance of such a device is that it will provide a crucial background forcing theoreticians to keep their theories grounded: if you have a theory, it had better agree with the newest experimental observations.

We should ideally have both experiments and theories. The Standard Model itself contains more than 20 funda- mental parameters, constants of nature, that are begging for an explanation. The masses of the fermions, known also as matter particles, are ascribed to the Brout-Englert-Higgs mechanism, just as the mass of the Higgs particle itself. Yet the Brout-Englert-Higgs theory does not explain why the postulated coupling parameters are such that they generate the mass spectrum that has been measured, numbers that vary wildly. Other interaction parameters-those of the gauge fields, due to the so-called force-carrying particles-can be explained to some extent, though most details of their origin are also mysterious. The relative strengths of the force couplings hint toward a further unification at extremely high energies, even though no predictions arising from this idea have been verified. There is definitely something that we have not understood.

A notorious and brave approach is called superstring theory. This approach has not led to new predictionsapart from those that are unverified by observations, such as the prediction of supersymmetry at LHC energies. Superstring theory has been criticized for this failure, but it must be emphasized that other approaches achieved far less. We can note that, although the Standard Model was not explained at all, string theory does lead to a zoo of particles that are not so unlike those of the Standard Model, and many of its practitioners interpret this fact as an encouragement to reinforce their efforts. All I can add is that the more we keep staring at the Standard Model's parameters, the more it seems that natural explanations should be asked for, and the existing theories do not seem to converge toward answers.

It is here that I suggest looking at the quantum-mechanical nature of all observed phenomena. Superstring theory has not provided any explanation as to what quantum mechanics is about. None of today's cherished theories are providing any evidence for conceivable constraints on the quantum-mechanical nature of their equations.

Imagine that further scrutinizing what is called quantum mechanics could lead to new ideas. Perhaps there are things that physicists do not yet see, but that we can still speculate about. Let me briefly remind the reader about the developments in quantum mechanics that got us entwined in a messy knot of problems. Back in 1932, John von Neumann gave a so-called proof of the statement that quantum mechanics cannot be explained in terms of hidden degrees of freedom, which would restore some deterministic interpretation of Schrödinger's wave function. ${ }^{7}$ His proof was later dismissed. Both the proof and its dismissal showed that the authors understood the mathematical nature of the equation, but missed the point that the physical world might be much more complex than just this one abstract equation, in which all sorts of possible complications were suppressed.

A very important paper was written by Albert Einstein, Boris Podolsky, and Nathan Rosen, arguing that indeed some essential ingredient was missing in this quantum 
theory. ${ }^{8}$ When considering an atom emitting two photons at once, quantum mechanics suggests that we can measure the position of one photon and the momentum of the other photon, but since the two photons are equal-entangled, to be precise-these measurements together should provide information for both photons that would be forbidden by the same quantum theory.

In 1952, David Bohm took an original idea of Louis de Broglie literally: the wave function acts as a pilot, telling the particle where to go. ${ }^{9}$

John S. Bell took up the same question, but insisted on restoring the notion of locality and causality in the interpretation of the wave function; both Bohm's theory and the Einstein-Podolsky-Rosen argument describe the events while giving up the notion of locality. ${ }^{10}$ Bell tried to rephrase everything in a powerful theorem, which would become famous. Starting from assumptions that he considered to be utterly reasonable, he derived this principle: you cannot build a theory of hidden variables that explains what Schrödinger's equation describes if you insist that it be local.

A different avenue was the argument that the wave function displays everything that might be happening, including all alternatives: an infinite set of different universes can all be evolving, together forming an even grander body later called the multiverse, or omniverse. The multiverse would be like a gigantic tree, every branch, every leaf of it being an entire universe. This many-worlds theory was formulated by Hugh Everett and strongly advocated by Bryce DeWitt. ${ }^{11}$ The theory would not change the equations, and basically, it would take for granted that quantum mechanics can never predict what will happen, beyond the level of statistical statements. You will always be surprised as to which world you apparently entered into, while, with different probabilities, all other possible worlds are realized somewhere else in the multiverse.

I think they are all mistaken. What the Schrödinger equation is describing is not exactly what is happening; it merely describes the tip of a gigantic iceberg, in which most processes happen far beneath the waterline. All that needs to be realized is that, like in any scientific theory, predictions concerning the outcomes of experiments cannot be expected to be infinitely accurate. There are margins of error, due to the fact that we have been using statistics. The statistical assumptions that went into Schrödinger's equations concern variables that give shape to space and time, fluctuating far too fast to be included in such a way that we can control them. We don't control them and this is the reason why we discover that our predictions come with margins of error, like in any other scientific theory. I think that the internal mathematical nature of Schrödinger's equation supports this view, but there is too much unknown at present, so that building models for what really happens is still difficult.

This idea would be covered in hidden variable theories. But hidden variables are now far more sophisticated than what earlier researchers had been thinking of-I will return to the consequences of our use of statistics shortly.

Some of my readers will object that Bell did emphatically include such hidden variables in his treatment, but he made assumptions that need not be valid. Indeed, what von Neumann had done, and what Bell did later, was to construct a no-go theorem. They both badly wanted to construct a hidden variable theory, but when they did not succeed, they decided to close the lid for good, that is, to prove that such a theory cannot be constructed. Time and again, however, the history of science has shown that no-go theorems do not say much more than that the avenues inspected by their authors do not lead to a desired result, so that, all that should have been done was to modify the assumptions.

To obtain a more solid understanding of what quantum mechanics really reveals, we have to go back a long way into the history of physics. What happened was that long sequences of discoveries not only enriched our knowledge tremendously, they also narrowed our way of thinking, so much so that some obvious facts are now considered to be obsolete, just because, in practice, our streamlined vision has become much more powerful. Take for instance the way we describe a moving object. Real numbers are used to describe positions, velocities, energies, and so on. What are real numbers? They are a natural generalization of rational numbers. Rational numbers are numbers possible to arrive at if an integer is divided by another integer. Integers are simply numbers that are used in counting, for instance the number of steps needed to get across a room. Integers can be stored in a computer, but before that we have to realize that there are limits to how far we can count, in practice. The simplest integers are called bytes. They can vary from 0 to 255 but no further than that. Each byte can be expressed in terms of eight bits. Bits can take only two values, which may be called 0 and 1 , or alternatively, + and -.

Those real numbers used now are so powerful that it is easy to forget that they are manmade. Every real number that we use to describe our world would require an infinite amount of computer space to store it, because it contains an infinite sequence of digits, all of which can be arbitrary. Is this really the correct way to indicate distances, velocities, and so on? It could be that all positions and all velocities eventually only require integers to specify their values. This would mean that particles live in a space-time that is not strictly continuous but could be what is called a lattice.

I am not suggesting that I know for sure that spacetime is a lattice. I don't. But I do know that possibilities like this would profoundly change the ways one could think of a particle, and with that, the way we should formulate the laws of physics they obey. In practice it is very hard to formulate laws of physics for lattice-particles that fit well with the tremendously sophisticated sci- 
ence that exists today. Schrödinger's beautiful equation seems to be what we get if we want to describe particles and at the same time hang on to those somewhat artificial real numbers.

There is another aspect of modern science that has become so self-evident that researchers fail to notice that the deeper physical laws could deviate from standard dogma. It is called statistics. Quantum mechanics provides ways to estimate very precisely the statistical behavior of large numbers of particles, or large numbers of experiments, or large numbers of observers. When you consider a large reservoir containing molecules or other particles moving chaotically, then it is only too tempting to say that the whole lot of them will be fundamentally unpredictable when we ask for all the details of the motion of every one of the particles individually. There will be exceptional chance events, and they will be fundamentally unpredictable, with or without quantum mechanics.

To formulate his theorem, Bell needed to introduce two hypothetical observers, Alice and Bob. Both Alice and Bob had to choose what they would measure. They had the free will to decide what to choose. But if an omnipotent theory prescribes every move with infinite precision, such free will does not exist. Nobody today thinks of physical laws that also control the outcomes of arbitrary, individual measurements in a gas consisting of billions of particles, but that is what we should try to find. Note that I am not pretending that a theory can be found that predicts where every single atom or molecule will be going, but I do think that it is reasonable to suspect that laws of nature exist that would uniquely fix their behavior, even while our ability to predict will always be limited by our knowledge, our computation power, and our understanding.

To many of my readers-or the ones that may still be with me-what I just said sounds very much like letters we receive in our daily mail from amateur physicists. They are amateurs because they usually exhibit a dismal lack of knowledge and understanding of modern science. Like many of my colleagues, I often quickly discard such letters, but sometimes they are fun to read. More to the point, by not knowing how our world has been found to hang together, their authors could have stumbled into some independent ways of asking questions. Indeed, when I think of questions concerning quantum mechanics, I know I have to make giant leaps of logic. Often, these are giant leaps backward. What I really want is to use kinds of logic that do not make use of conventional number systems, or other conventional ways to describe our understanding of physical laws. But then, before irritating my colleagues with my crackpot findings, I search for the proper connections with real science. This is because I do know what conventional science says about quantum mechanics, particles, and number systems. And today I think of ways to attach unconventional views to the beautiful edifice known as the Standard Model.
This is the reason why the discussion about quantum mechanics may be an important discussion after all. We cannot accept a theory that, in the very end, only predicts statistical properties of the small particles, but leaves them free, to some extent, to do whatever they like. If we dig deep enough, we should find some superior scenario of the dynamical laws, and this new scenario must carry implications for the way we build models.

The Standard Model does not pose any restrictions concerning the quantum equations on which it is based, but it might be what is needed to make the next step in particle theory. Today, the known elementary particles form a bunch of objects with masses ranging from a few milli-electron volts to somewhere around a $\mathrm{TeV}$, a stretch of some 15 orders of magnitude. Particles heavier than a few TeV cannot be observed with the machines we have today. There is no fundamental limit to the mass of a particle until we reach the Planck mass, some 16 orders of magnitude beyond where we are now. It is the meshes of our lattice that forbid elementary objects beyond that. This is my iceberg: only one part in $10^{16}$ is visible above the waterline.

The particles currently hidden from view would undergo interactions and oscillations that are so much faster as time proceeds, that we may call them fast hidden variables. Today's Schrödinger equation has all fast variables suppressed; we cannot detect such fast phenomena. Now, I claim that the fast, invisible phenomena could be described by classical laws, but these movements are fundamentally unobservable, and we can only deal with what we see. In a way invisible to us now, the things we see are controlled by fast-moving particles, particles that form the bulk of the iceberg. Today's science yields an equation that can be used to evade those particles, but this has come at a price. We have hit upon unpredictable behavior that should have been unsurprising: these are the whims of the hidden variables. To my taste, this way to interpret what the quantum mechanical laws are about sounds much more reasonable than assumptions concerning pilot waves or multiverses. But most importantly, any reference to statistical averaging should be banned from the initial assumptions because these indeed lead to no-go theorems such as Bell's. Experimenters do not have the free will to do something that is not controlled by deterministic laws of nature.

When making such claims, one has to come with mathematical models. The models in use today are imperfect, but encouraging indications exist that the Schrödinger equation is exactly what one should expect from such hidden physical processes. ${ }^{12}$

I found that it may be important to make yet another step backward: we have become used to the utility of complex numbers to describe quantum wave functions. Why? Some people claim it to be one of those quantum peculiarities, setting quantum mechanics aside from all classical theories, even though complex numbers have often also 
proved useful for calculations in classical topics. What is a complex number? One of the marvels of modern science is that we learned how to take the square root of negative real numbers. But then here also, understanding is often replaced by mysticism.

This is when one forgets how complex numbers are defined. Complex numbers are simply pairs of real numbers, with rules attached for how to add, subtract, multiply, and divide such pairs of numbers. In quantum theories, one is tempted to forget what this means: the physical state of a system includes one or more objects that can come in two forms, represented by the two components of the complex function that represents a wave function. One can also decide to describe such components in a more direct manner, avoiding the use of complex quantities, which after all, are also manmade inventions. Such a direct language may lead to some useful insights. When restricting ourselves to real wave functions only, one finds that the quantum Hamiltonian is an antisymmetric matrix. Finding eigenvectors and eigenvalues of matrices is common practice for easing calculations, but here, antisymmetry would force us to split the states into pairs again, leading us straight back to those useful complex numbers.

The mathematical insights gained through this brief excursion may turn out to be useful. Quantum mechanical models with a deeper, deterministic basis cannot support the use of arbitrary real numbers as input parameters. This means that constants such as the masses and coupling strengths of particles cannot be assumed to be any real number. In some intricate way, these must be expressed in terms of integer numbers only. This suggests that one might hit upon special types of field theories under which the input parameters can be calculated.

\section{Gerard 't Hooft}

\section{Sheldon Lee Glashow replies:}

Gerard 't Hooft acknowledges that we have strong evidence supporting the validity of the Standard Model up to the $\mathrm{TeV}$ domain, but laments that we do not "understand why this model would be all there is to describe what happens beyond that scale." For this reason, he supports the construction of a far more powerful hadron collider so as to access center-of-mass energies of $100 \mathrm{TeV}$. More and more particles may or may not be observed, but whatever we find at higher energies,

the more we keep staring at the Standard Model's [many] parameters, the more it seems that natural explanations should be asked for, and the existing theories [including string theories] do not seem to converge to answers.

't Hooft suspects that the answers may lie at the very root of the problem, our incomplete understanding of quantum mechanics. He recalls the puzzle of Einstein's spooky action at a distance, briefly mentioning the De Broglie-Bohm pilot wave theory, Bell's theorem, and Hugh Everett's many-worlds interpretation of quantum mechanics. "I think they are all mistaken," he writes. Real numbers are 't Hooft's culprits.

"Schrödinger's beautiful equation," 't Hooft writes, "seems to be what we get if we want to describe particles and at the same time hang on to those somewhat artificial real numbers." He is not the first to question the reality of real numbers. Years ago, in the context of mathematical physics, Gregory Chaitin asked, "How real are real numbers?"13 More recently, Nicolas Gisin found that

real numbers are marvelous tools that should not be abandoned. However, their practical use should not blind the physicists; after all, their use does not force us to believe that "real numbers are really real."

Both authors point out that no measured numbers can be real numbers, because all are subject to finite experimental error. They, like 't Hooft, imagine there to be an underlying digital or lattice structure to nature. 't Hooft is hoping to do more than merely imagine. He strives to create a deterministic version of quantum mechanics that accounts for the "fast hidden variables" lying below the waterline and forever beyond accessible energies. He would attach such "unconventionalviews to the beautifuledificeknown as the Standard Model." Whether he can succeed in such an ambitious endeavor I cannot say, but he has certainly earned the right to try.

\section{Gerard 't Hooft is a Nobel Laureate and Professor of Theoretical Physics at Utrecht University.}

Sheldon Lee Glashow is a Nobel Laureate, Higgins Professor of Physics, emeritus, at Harvard University, and University Professor, emeritus, at Boston University.

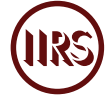

1. Max Planck, "Zur Theorie des Gesetzes der Energieverteilung im Normalspectrum," Verhandlungen der Deutschen Physikalischen Gesellschaft 2 (1900): 237. English translation, "On the Theory of the Energy Distribution Law of the Normal Spectrum," in The Old Quantum Theory, ed. Dirk ter Haar (Oxford: Pergamon Press, 1967), 82.

2. Planck, letter to Robert Williams Wood, October 7, 1931.

3. Abraham Pais, Inward Bound: Of Matter and Forces in the Physical World (Oxford: Oxford University Press, 1986).

4. Erwin Schrödinger, "An Undulatory Theory of the Mechanics of Atoms and Molecules," Physical Review 28, no. 6 (1926): 1,049, doi:10.1103/PhysRev.28.1049. 
5. Max Born, "Zur Quantenmechanik der Stoßvorgänge (Toward the Quantum Mechanics of Collision Processes)," Zeitschrift für Physik 38, no. 11-12 (1926): 803-27.

6. Nicolaas Godfried van Kampen, "Ten Theorems about Quantum Mechanical Measurements," Physica A: Statistical Mechanics and its Applications 153 (1988): 97-113, doi:10.1016/0378-4371(88)90105-7.

7. John von Neumann, Mathematical Foundations of Quantum Mechanics: New Edition (Princeton: Princeton University Press, 2018).

8. Albert Einstein, Boris Podolsky, and Nathan Rosen, "Can Quantum Mechanical Description of Physical Reality be Considered Complete?" Physical Review 47 (1935): 777, doi:10.1103/PhysRev.47.777; Max Jammer, The Conceptual Development of Quantum Mechanics (New York: McGrawHill, 1966).

9. David Bohm, "A Suggested Interpretation of the Quantum
Theory in Terms of 'Hidden' Variables, I,' Physical Review 85, no. 2 (1952): 166-79, doi:10.1103/PhysRev.85.166.

10. John Stewart Bell, "On the Einstein Podolsky Rosen Paradox,” Physics 1 (1964): 195, doi:10.1103/PhysicsPhysiqueFizika.1.195.

11. Hugh Everett, "Relative State Formulation of Quantum Mechanics," Reviews of Modern Physics 29 (1957): 454, doi:10.1103/RevModPhys.29.454; Bryce DeWitt, in The ManyWorlds Interpretation of Quantum Mechanics, ed. DeWitt and Neill Graham (Princeton: Princeton University Press, 1973).

12. Gerard 't Hooft, "Deterministic Quantum Mechanics: The Mathematical Equations" (2020), arXiv:2005.06374.

13. Gregory Chaitin, "How Real Are Real Numbers?” (2004), arXiv:math/0411418v3, doi:10.1590/s0100-60452011000100006.

14. Nicolas Gisin, "Classical and Intuitionistic Mathematical Languages Shape Our Understanding of Time in Physics," Nature Physics 16, (2020): 114-16, doi:10.1038/s41567-019-0748-5. 